\title{
Leveraging the Niche of Open Data for Disease Surveillance and Health Education
}

\author{
Ta-Chien Chan*, Yung-Chu Teng, Chiao-ling Kuo, Yao-Hsien Yeh and Bo-Cheng Lin \\ Academia Sinica, Taipei City, Taiwan
}

\section{Objective}

To visualize the incidence of notifiable infectious diseases spatially and interactively, we aimed to provide a friendly interface to access local epidemic information based on open data for health professionals and the public.

\section{Introduction}

Transparency of information on infectious disease epidemics is crucial for not only public health workers but also the residents in the communities. Traditionally, disease control departments created official websites for displaying disease maps or epi-curves with the confirmed case counts. The websites were usually very formal and static, without interaction, animation, or even the aid of spatial statistics. Therefore, we tried to take advantage of open data and use a lightweight programming language, JavaScript, to create an interactive website, named "Taiwan Infectious Disease Map (http://ide.geohealth.tw/)“. With the website, we expect to provide real-time incidence information and related epidemiological features using interactive maps and charts.

\section{Methods}

This study used infectious-disease-related open data from Taiwan's open data platform (http://data.gov.tw) maintained by the Taiwan CDC. It covers 70 types of infectious diseases starting from 2004, and the latest status is updated every day. We then automatically bridge this data into our database and calculate the age-adjusted incidence rate by annual census data and $2000 \mathrm{WH} 0$ standard population. The spatial resolution is mostly at the township level, except that resolution for sexually-transmitted infectious diseases is at the city level. The temporal resolution is month and year, except for dengue fever, which is by week.

We used R software to automatically compute incidence every day, and also used its package named "spdep" to compute the spatial clusters of the selected infectious diseases online. In addition, we used JavaScript language, PHP, OpenLayers 3 and Highcharts to implement interactive maps and charts. All the data and graphical figures from the charts viewed in this website can be downloaded freely. The temporal animation slider can be played and paused at any time point. The health education button can directly link to an introduction to the selected infectious disease maintained by the Taiwan CDC.

\section{Results}

The website of the Taiwan Infectious Disease Map is displayed in Figure 1. The users can select the temporal precision, types of infectious diseases, spatial precision and the gender at the beginning. In this case, the left map is the spatial distribution of the cumulative incidence of tuberculosis (TB) in 2016. The darker red color represents higher incidence. The right top panel is the ranking of TB incidence among 368 townships. The right middle panel is the ranking of TB incidence among 22 cities or counties. The right bottom panel is the annual TB incidence from 2004 to the current date. The highest TB incidence was 67.47 per 100,000 in 2004, and this declined sharply to 15.92 per 100,000 in 2015 .

\section{Conclusions}

With this user-friendly web application, the public and local public health workers can easily understand the current risk for their townships. The application can provide relevant health education for the public to understand diseases and how to protect themselves. The spatial clusters, gender distribution, age distribution, epi-curve and top ten infectious diseases are all practical and important information provided from this website to assist in preventing and mitigating next epidemic.

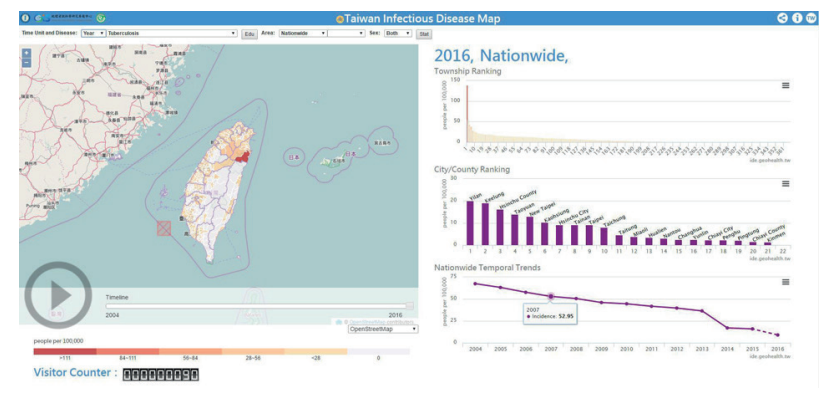

\section{Keywords}

Infectious disease; standardized incidence; spatial visualization; spatial clustering

\section{Acknowledgments}

This research was supported by a grant from Academia Sinica (Multidisciplinary Health Cloud Research Project: Technology Development and Application of Big Health Data).

\section{*Ta-Chien Chan}

E-mail: dachianpig@gmail.com 